\title{
Dental whitening effect of an oral probiotic
}

\author{
Jeffrey D. Hillman ${ }^{1}$, Emily McDonell ${ }^{1}$, Charles H. Hillman ${ }^{2}$ and Martin Handfield ${ }^{1 *}$ \\ ${ }^{1}$ Oragenics, Inc., 13700 Progress Blvd., Alachua, FL 32615, USA \\ ${ }^{2}$ University of Illinois, Department of Kinesiology and Community Health Department, Champaign, IL 61801, USA
}

\begin{abstract}
Aim: Oral care probiotics that incorporate hydrogen peroxide-producing strains of Streptococcus oralis may represent an effective means of naturally whitening teeth, when whitening is indicated. The purpose of this study was to evaluate the cosmetic benefit ("whitening effect") of a laboratory culture of $S$. oralis strain KJ $3^{\circledR}$ and further investigate its mechanism of action.

Methods: Ceramic discs were stained with several agents and subsequently treated with live suspension of peroxide-producing bacterial cultures over a period of 4 weeks. Colorimetric changes in the lightness value of the stained ceramic disks were measured at weekly intervals during the treatment phase.

Results: At the conclusion of the 4-week experimental period, a statistically significant whitening effect was observed from the 2-week point through week 4. The whitening effect was influenced by the presence of glucose and/or catalase in the incubation medium. Interestingly, this whitening effect observed did not plateau at four weeks, indicating that maximum dental whitening effect of the $S$. oralis strain had not occurred within the timeframe of the study.

Conclusion: The results obtained herein support the hypothesis that a $S$. oralis strain may be able to significantly lighten stained dental ceramic disks, which may provide scientific support to the whitening effect of commercially available probiotic formulations that include $S$. oralis $\mathrm{KJ} 3^{\circledR}$.
\end{abstract}

\section{Introduction}

The use of tooth whitening techniques to improve the esthetics of the dentition has increased dramatically during the past two decades [1]. Tooth whitening procedures are typically based either on the use of peroxide bleaching agents to address stains below the tooth surface or abrasive agents to remove surface tooth stains. Tooth discoloration varies in etiology and appearance. While lifestyle and dietary choices as drinking coffee, tea, red wine and fruit juice, and utilizing tobacco products can produce tooth staining or discoloration [2,3], pulpitis or trauma to the teeth, for example, can also cause tooth discoloration. Professional scaling and polishing of teeth or the use of whitening toothpastes can help to remove many types of surface stains, primarily through the action of their abrasive/polishing agents, when indicated. However, for difficult sub-surface discoloration, peroxide bleaching methods have proven to be effective in whitening the treated tooth surfaces. These bleaching methods are based upon hydrogen peroxide applied directly to the tooth surface, or produced by a chemical reaction from carbamide peroxide or sodium perborate [4]. Peroxide bleaching methods may be employed by a dental professional during a single or multiple visits, or by an individual using a special mouth tray or plastic polymer strip, loaded with a peroxide agent, over a treatment period that can extend for weeks or possibly months. The success of a bleaching procedure depends on several factors, including the concentration of the peroxide agent, the ability of the agent to reach the stain-causing entity, and the duration and frequency that the peroxide agent is applied to the stained tooth surfaces. A large number of at-home tooth whitening products are available today and include toothpastes, mouth rinses, and chewing gums, as well as products that contain a peroxide bleaching agent. These peroxide products typically consist either of trays, custom made by a dental professional, and filled with from 5 to $22 \%$ solution of carbamide peroxide, or over-the- counter (OTC) products containing carbamide peroxide or hydrogen peroxide in a pre-fabricated tray or in whitening strips [5].

The most common side effects for bleaching procedures on vital teeth include transient tooth sensitivity and gingival irritation. Tooth sensitivity resulting from bleaching can generally be managed by bleaching less frequently or reducing the concentration of the bleaching agent or shortening the application time [6,7]. Tooth sensitivity will typically disappear shortly after the bleaching process is terminated. Another common side effect of bleaching procedures is soft tissue irritation. This effect most commonly is caused by a poor-fitting bleaching tray or by applying an excessive amount of bleaching agent to the tray [6]. In some instances, significant damage to enamel has been reported as a result of long-term or inappropriate use of homecare bleaching products $[8,9]$. It is generally recognized that many of the side effects noted above can be mitigated by professionally applied bleaching protocols, where prolonged whitening effects with minimum side effects are typically obtained, due in part to new technology and to the proper isolation methods that are followed during in-office bleaching.

Recently, a probiotic cocktail that can be delivered in several modalities has been developed for use in maintaining oral health [1012]. This oral care probiotic called ProBiora $3^{\circledR}$ contains a cocktail of

Correspondence to: Martin Handfield, MS, PhD, Oragenics, Inc. 13700 Progress Blvd., Alachua, FL 32615, USA, Tel: 386-418-4018 ext. 241; Fax: 386-418-1660; E-mail: mhandfield@oragenics.com

Key words: probiotic, teeth whitening, peroxide-producing bacteria, dental esthetics, cosmetic dentistry

Received: January 14, 2016; Accepted: February 12, 2016; Published: February 17,2016 
three strains of naturally occurring oral bacteria, each with a specific function of maintaining a healthy oral environment. The probiotic strains consist of Streptococcus oralis $\mathrm{KJ}^{\circ}$, Streptococcus uberis $\mathrm{KJ}^{\circ}$, and Streptococcus rattus $\mathrm{JH} 145^{\circ}$. Interestingly, the S. oralis strain and, to lesser degree, the $S$. uberis strain produce hydrogen peroxide, and the mechanism of antagonistic activity against periodontal pathogens has been demonstrated to depend on this metabolic activity $[13,14]$. Because of hydrogen peroxide production by S. oralis and S. uberis, it was proposed that the probiotic cocktail may have an additional cosmetic benefit of naturally whitening teeth over a treatment period of weeks or months. The present study was designed to test this hypothesis in vitro by exposing stained dental ceramic disks daily to fresh suspensions of S. oralis KJ3 over a period of 4 weeks and monitoring the effectiveness of the treatment to whiten the stained ceramic disks.

\section{Material and methods}

\section{Whitening}

Ten dental ceramic disks (courtesy of Dr. Kenneth J. Anusavice, University of Florida, Gainesville, FL) were stained over an eight-week period with tea (Lipton) and chlorhexidine $(0.12 \%$, Hi Tech Phamacal Co., Amityville, NY). Each disk was placed in a $50 \mathrm{ml}$ conical (Falcon) plastic test tube. Three $\mathrm{ml}$ of brewed tea (prepared by the addition of a family size tea bag to $200 \mathrm{ml}$ of boiling water for $5 \mathrm{~min}$ ) were added to cover the disks. After 24 hours of incubation at room temperature, the tea was removed by decanting, the disk was rinsed with $5 \mathrm{ml}$ of tap water, and the tea solution was replaced with $3 \mathrm{ml}$ of a commercial $0.12 \%$ chlorhexidine mouth rinse for 24 hours. Steps 2 and 3 were repeated for 4 weeks, Monday through Friday, and the disks remained in Friday's solution over the weekend. The surface color of the stained disks was quantitatively measured using a Chroma Meter CR-400 colorimeter (Minolta, Ramsey, NJ). The color or lightness values for the stained disks were generated directly by placing the instrument's measuring head over the disks. Standard color plates were used to calibrate the colorimeter. The treatment phase began one day after the final colorimeter readings were taken. S. oralis $\mathrm{KJ} 3 \mathrm{sm}$ is a naturally occurring genetic variant of $\mathrm{KJ} 3$ that is resistant to $1 \mathrm{mg} / \mathrm{mL}$ of streptomycin [15]. Nine separate cultures of KJ3sm, inoculated from starter plates, were grown in $30 \mathrm{ml}$ of Todd Hewitt Broth (Becton, Dickinson Co., Sparks, MD) supplemented with $0.1 \%$ sodium bicarbonate $/ 0.5 \%$ glucose $/ 0.1 \%$ streptomycin sulfate in an environmental shaker $(200 \mathrm{rpm})$ at $37^{\circ} \mathrm{C}$. After overnight incubation, the cells were harvested by centrifugation at room temperature, washed once with $10 \mathrm{ml}$ of Amies media (Amies 1967), and resuspended in $30 \mathrm{ml}$ of fresh Amies media with or without glucose (Sigma-Aldrich, Inc., St. Louis, MO) and catalase at a concentration of $1000 \mathrm{U} / \mathrm{ml}$ (Sigma-Aldrich, Inc.) as shown in Table 1. The Amies transport media was formulated to maintain the viability of microorganisms without significantly supporting their growth [16]. A medium control contained $30 \mathrm{ml}$ of Amies medium with glucose

Table 1.Treatment Conditions Used in the Current Protocol.

\begin{tabular}{|c|c|c|c|c|}
\hline \multirow[t]{2}{*}{ Group } & \multirow{2}{*}{$\begin{array}{c}\text { Disk } \\
\text { Number }\end{array}$} & \multicolumn{3}{|c|}{ Treatment Conditions } \\
\hline & & $\begin{array}{c}\text { KJ3ssm } \\
\left(\sim 10^{9} \mathrm{cfu} / \mathrm{ml}\right)\end{array}$ & $\begin{array}{c}\text { Glucose } \\
(0.5 \%)\end{array}$ & $\begin{array}{c}\text { Catalase } \\
(3,000 \mathrm{U} / \mathrm{ml})\end{array}$ \\
\hline $\mathrm{A}^{1}$ & $1,2,3$ & + & + & Inactivated \\
\hline $\mathrm{B}^{2}$ & $4,5,6$ & + & + & Active \\
\hline $\mathrm{C}^{3}$ & $7,8,9$ & + & - & Inactivated \\
\hline $\mathrm{D}^{4}$ & 10 & - & + & Inactivated \\
\hline
\end{tabular}

${ }^{1}$ Experimental

${ }^{2}$ Catalase control

${ }^{3}$ Glucose control

${ }^{4}$ Medium control and inactivated catalase but no cells. Where indicated, catalase was inactivated by heating in a boiling water bath for 5 minutes. The entire $30 \mathrm{ml}$ aliquots described in step 2 and Figure 1 were added to $50 \mathrm{ml}$ Falcon tubes containing 1 stained dental ceramic disk per tube and incubated at $37^{\circ} \mathrm{C}$ for $24 \mathrm{~h}$. These treatment steps were repeated daily, Monday through Friday, for 4 weeks, and the disks remained in Friday's solution over the weekend. The lightness value for each stained disk was measured weekly during the treatment phase, which was carried out over a 4 -week period.

\section{Statistical analysis}

The $\mathrm{L}$ value (lightness) was plotted as a function of time using the mean group values $( \pm$ S.D.). Inter- and intra-group $L$ values were compared for statistically significant differences. A 4 (Treatments: A, B, C, D) x 5 (Time: Weeks 0, 1, 2, 3, 4) mixed-model repeated measures ANOVA was conducted with Greenhouse-Geisser correction to determine the differential influence of various treatments on $L$ values across time. Follow-up analyses determined treatment effects at each time point. Post hoc Bonferroni-corrected t tests were used to determine significant differences among the treatments. All analyses used a family-wise alpha level after Bonferroni correction of $p=0.05$.

\section{Results}

The $\mathrm{L}$ value (lightness) was plotted as a function of time using the mean group values ( \pm S.D.; Figure 1). The trend line for Group A (experimental group) data had a slope of 2.37 , which was substantially greater than the slopes of the trend lines for Group B (catalase control group; slope, -0.54), Group C (glucose control group; slope, 0.52 ) and Group D (medium control group; slope, -0.84), indicating that disks in Group A were becoming lighter or whiter over time at a much faster rate than the disks in the other groups. At the conclusion of the 4-week experimental period, a lightening of the ceramic disks in Group A was readily apparent to the unaided eye (Figure 2). The data was further analyzed as follows: $S$. oralis, strain $\mathrm{KJ} 3 \mathrm{sm}$, incubated in the presence of glucose and air (Group A) produced a significantly larger whitening effect on tea and chlorhexidine-stained ceramic disks at Weeks 2-4 than Control Groups B and D, and a significantly larger effect than Control Group C, at Week 4. Inclusion of catalase in the medium

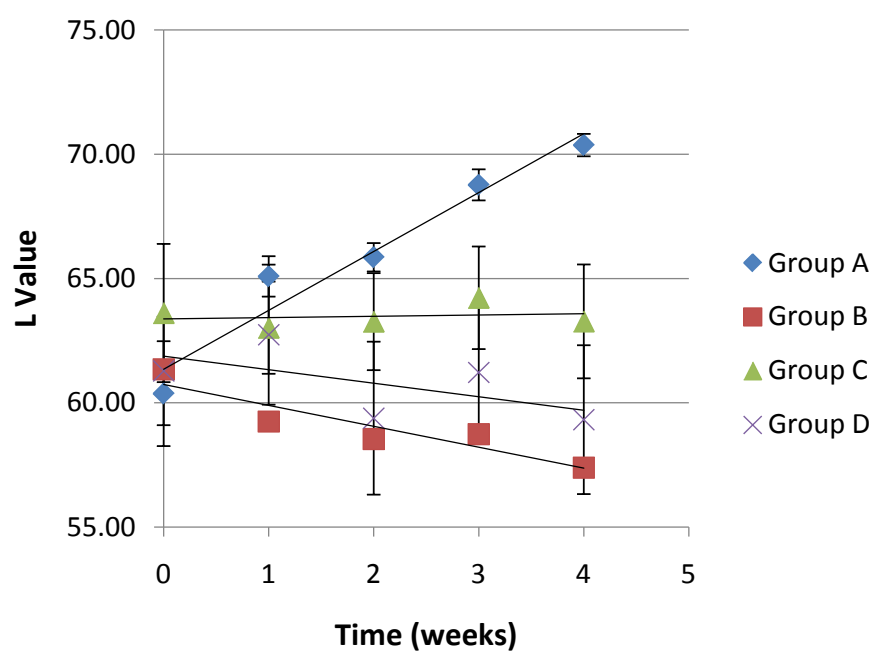

Figure 1. Impact of an oral probiotic mixture on stained disks whiteness (L-value), as a function of duration of treatment. The experimental design and corresponding treatment groups are presented in Table 1. Group A is the experimental group, and Groups B-D are controls. An increase in L-value corresponds to an increase in whiteness. Several of the disks used are presented in Figure 2. 


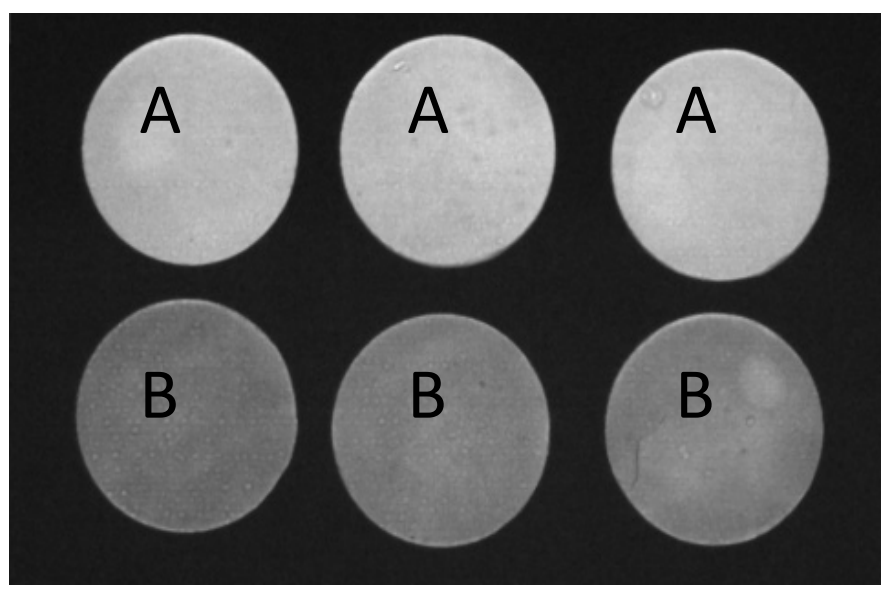

Figure 2. Stained ceramic disks used in that study at 4 weeks after the probiotic treatment initiation. The experimental design and corresponding treatment groups are presented in Table 1. Group A is the experimental group and Group B, the catalase negative control.

(Group B), to enzymatically destroy hydrogen peroxide generated by $\mathrm{KJ} 3 \mathrm{sm}$, significantly reduced the whitening effect of KJ $3 \mathrm{sm}$. When glucose was omitted from the incubation medium (Group C), a smaller whitening effect was observed than in Group A, and this difference became significant at the 4 -week point. Medium supplemented with glucose and inactivated catalase (Group D) showed no tendency to produce lightening of the stained ceramic disks. The plot of $\mathrm{L}$ value as a function of time for Group A in Figure 1 did not plateau, indicating that maximum whitening effect from exposure of stained disks to the KJ3sm strain had not occurred within the timeframe of the study.

\section{Discussion}

Over-the-counter (OTC) products are currently available that utilize carbamide peroxide or hydrogen peroxide either in prefabricated or user-modified trays or incorporated into strips that users apply to their teeth $[5,6]$. This cosmetic procedure, however, is not currently recommended for everyone, and is not without its side effects [7,17-19]. Tooth sensitivity is the most common side-effect of bleaching, observed in $15 \%$ to $78 \%$ of the patients $[4,20]$. Gingival irritation during treatment is another commonly reported side effect $[17,20]$, which can be mitigated by using appropriate in-office isolation protocols. In animal toxicity studies, it was reported that exposure of the gingiva to $1 \%$ hydrogen peroxide for 6 to 48 hours resulted in epithelial damage and acute inflammation of the subepithelial connective tissue, and long-term application of $3 \%$ hydrogen peroxide in the hamster cheek pouch twice weekly resulted in inflammatory changes $[21,22]$. Furthermore, surface alterations in enamel topography were routinely observed following tooth bleaching with $10 \%$ and higher concentrations of carbamide peroxide [23-25]. If we consider the above potential side effects of bleaching protocols, it is reasonable to conclude that preparations with the lowest concentration of a bleaching agent would provide the maximum level of short and long-term safety for the user. Furthermore, since considerable care must be exercised by the user when self-applying bleaching trays and strips to teeth at home, time and effort would likely be minimized with the use of a more userfriendly delivery vehicle. It was hypothesized by the authors that the use of indigenous oral bacterial strains that are known to metabolically produce hydrogen peroxide, when delivered to the surfaces of teeth in a convenient mouthwash format, may represent a natural alternative for whitening teeth. There are a number of non-pathogenic bacterial species that are known to produce hydrogen peroxide, including lactobacillus, bifidobacterium and viridans streptococcus strains [26,27].

Streptococcus oralis $\mathrm{KJ} 3 \mathrm{sm}$ was selected for testing in this in vitro whitening model because it has been shown in a clinical trial to be safe and well tolerated, with no user complaints of tooth sensitivity or gingival irritation throughout the 8-week treatment period involving twice daily product use $[11,12]$. Furthermore, this strain has been reported to inhibit the growth of microorganisms known to be associated with periodontal diseases, and is an excellent hydrogen peroxide producer $[14,15,28]$. There is mounting clinical evidence to support the efficacy of probiotic formulations that contain Streptococcus oralis KJ3sm. In one study, a probiotic mouthwash that contains a cocktail of $S$. oralis KJ3sm combined with S. uberis KJ2sm and S. rattus JH145 (formulated together in ProBiora $3^{\circ}$ ) was able to substantially affect the levels of dental pathogens in saliva and periodontal pathogens in subgingival plaque [12]. In an independently conducted randomized double-blind placebo-controlled clinical trial, the Caries Risk Test (CRT) values obtained with caries prone children were significantly affected by the use of ProBiora3 by decreasing the number of $S$. mutans and lactobacilli present in the salivary samples [30]. In another independently conducted clinical trial, 138 caries-prone children were treated with ProBiora 3 for one year and the prevalence and increment of initial and manifest caries lesions was examined at baseline and follow-up. This study demonstrated that the caries increment was significantly lower in the test group when compared with the placebo group $(\mathrm{p}<0.05)$, which suggested that early childhood caries development could be reduced through administration of probiotic chewing tablets containing ProBiora3 [31].

A colorimeter was employed in this in vitro study to quantitatively determine the extent of the whitening effect of $S$. oralis KJ $3 \mathrm{sm}$ under the various treatment conditions. A shade guide is typically used in clinical tooth whitening studies to measure the efficacy of a particular bleaching treatment. However, color matching using a shade guide can be subject to investigator variability and bias. Furthermore, the shade guide units are not evenly distributed in color space and are not as discriminating as the measured colorimeter parameters [29]. The results of the present study demonstrate that, in a laboratory model, the metabolically active, non-growing $S$. oralis $\mathrm{KJ} 3 \mathrm{sm}$ strain, is capable of significantly lightening a tea and chlorhexidine stained dental ceramic disk in a time-dependent fashion. Importantly, the lightening of the stained disks did not plateau over the course of the 4-week study, which suggests that the maximum dental whitening effect of the KJ3sm strain had not occurred within the time frame of the study, and that additional stain removal may be possible with longer treatment times.

The proposed mechanism for the observed whitening effect relates to the normal end product production of hydrogen peroxide by the KJ3sm strain. Peroxide is produced mainly from pyruvate by the pyruvate-oxidase activity in S. oralis, and it is excreted into the environment as an end product of sugar metabolism [15]. Hydrogen peroxide was shown in that study to be the mechanism used by S. oralis to inhibit the growth of periodontal pathogens. In the present study, the inclusion of active catalase in the incubation medium (Group B) demonstrated that hydrogen peroxide production is also the mechanism for the whitening effect observed with stained ceramic disks. Peroxide production, and thus the whitening effect, is dependent on the presence of a metabolizable carbon source (e.g., glucose). Thus, a whitening effect would not be expected when the incubation medium does not contain a carbon source. The small whitening effect observed when glucose was omitted from the incubation medium (Group C) may be explained by residual peroxide production resulting from metabolism 
of stored carbohydrate, most likely in the form of intracellular polysaccharide. The incubation medium itself (Group D) showed no tendency to cause lightening of the ceramic disks. The concentration of peroxide in contact with oral surfaces is much lower for a probioticbased whitening mouthwash than for the current range of peroxidebased whitening products. The whitening process, therefore, would be expected to be slower. It would, however, most certainly be safer to oral tissues, and more widely applicable to population groups that are currently contra-indicated for office and at-home tooth whitening procedures, when whitening is indicated. A probiotic-based whitening mouthwash also represents a more user-friendly delivery vehicle since it eliminates the need for using a mouth tray or polymer strip to limit contact of the whitening agent to only tooth surfaces.

\section{Conclusion}

The ProBiora3 mouthwash that incorporates three natural oral bacterial strains into its formulation, including the $S$. oralis $\mathrm{KJ} 3 \mathrm{sm}$ strain, has been clinically demonstrated to lower the levels of key dental and periodontal pathogens in the mouth $[11,12,30,31]$. This in vitro study suggests a further benefit of tooth whitening may be possible with the use of the ProBiora3 mouthwash containing hydrogen peroxide producing bacterial strains, and warrants conducting a properly powered human trial to quantify the extent of the whitening effect in clinical settings.

\section{Acknowledgments}

The Authors wish to thank Dr. Kenneth J. Anusavice (University of Florida, Ret.) for insightful discussions and for providing us with ceramic disks. The Authors would like to thank Dr. Robert Zahradnik (ret.) for his early contribution to this work.

\section{Disclosure}

The authors are disclosing that EM and MH were employees of Oragenics, a publically-traded company, at the time of submission of this manuscript. JDH (ret.) was previously employed by Oragenics. Drs. Jeffrey D. Hillman and Charles H. Hillman are father and son.

\section{References}

1. Sarrett DC (2002) Tooth whitening today. J Am Dent Assoc 133: 1535-1538. [Crossref]

2. Hattab FN, Qudeimat MA, al-Rimawi HS (1999) Dental discoloration: an overview. $J$ Esthet Dent 11: 291-310. [Crossref]

3. Watts A, Addy M (2001) Tooth discolouration and staining: a review of the literature. Br Dent 190: 309-316. [ Crossref]

4. Dahl JE, Pallesen U (2003) Tooth bleaching--a critical review of the biological aspects. Crit Rev Oral Biol Med 14: 292-304. [Crossref]

5. Gerlach RW, Gibb RD, Sagel PA (2000) A randomized clinical trial comparing a novel $5.3 \%$ hydrogen peroxide whitening strip to $10 \%, 15 \%$, and $20 \%$ carbamide peroxide tray-based bleaching systems. CompendContinEduc Dent Suppl 29: S22-S28. [Crossref]

6. Li Y (1996) Biological properties of peroxide-containing tooth whiteners. Food ChemToxicol 34: 887-904. [Crossref]

7. Haywood VB (2000) Current status of nightguard vital bleaching. CompendContinEduc Dent Suppl 28: S10-S17. [Crossref]

8. Cubbon T, Ore D (1991) Hard tissue and home tooth whiteners. CDS Rev 84: 32-35. [Crossref]

9. Hammel S (1998) Do-it-yourself tooth whitening is risky. US News and World Report:66.

10. Hillman JD, McDonell E, Hillman CH, Zahradnik RT, Soni MG (2009) Safety assessment of ProBiora3, a probiotic mouthwash: subchronic toxicity study in rats. Int J Toxicol 28: 357-367. [Crossref]
11. Hillman JD, McDonell E, Cramm T, Hillman CH, Zahradnik RT (2009) A spontaneous lactate dehydrogenase deficient mutant of Streptococcus rattus for use as a probiotic in the prevention of dental caries. J Appl Microbiol 107: 1551-1558. [Crossref]

12. Zahradnik RT, Magnusson I, Walker C, McDonell E, Hillman CH, et al. (2009) Preliminary assessment of safety and effectiveness in humans of ProBiora3, a probiotic mouthwash. J Appl Microbiol 107: 682-690. [Crossref]

13. Hillman JD, Socransky SS, Shivers M (1985)The relationships between streptococcal species and periodontopathic bacteria in human dental plaque. Arch Oral Biol 30: 791795. [Crossref]

14. Socransky SS, Haffajee AD, Dzink JL, Hillman JD (1988) Associations between microbial species in subgingival plaque samples. Oral Microbiol Immunol 3: 1-7. [Crossref]

15. Hillman JD, Shivers M (1988) Interaction between wild-type, mutant and revertant forms of the bacterium Streptococcus sanguis and the bacterium Actinobacillusactinomycetemcomitans in vitro and in the gnotobiotic rat. Arch Oral Biol 33: 395-401. [Crossref]

16. Amies CR (1967) A modified formula for the preparation of Stuart's Transport Medium. Can J Public Health 58: 296-300. [Crossref]

17. Leonard RH Jr, Haywood VB, Phillips C (1997) Risk factors for developing tooth sensitivity and gingival irritation associated with nightguard vital bleaching. Quintessence Int 28: 527-534. [Crossref]

18. Lee SS, Zhang W, Lee DH, Li Y (2005) Tooth whitening in children and adolescents: a literature review. Pediatr Dent 27: 362-368. [Crossref]

19. Tredwin CJ, Naik S, Lewis NJ, Scully C (2006) Hydrogen peroxide tooth-whitening (bleaching) products: review of adverse effects and safety issues. Br Dent J 200: 371376. [Crossref]

20. Tam L (1999) The safety of home bleaching techniques. J Can Dent Assoc 65: 453-455.

21. Martin JH, Bishop JG, Guentherman RH, Dorman HL (1968) Cellular response of gingiva to prolonged application of dilute hydrogen peroxide. J Periodontol 39: 208210. [Crossref]

22. Weitzman SA, Weitberg AB, Stossel TP, Schwartz J, Shklar G (1986) Effects of hydrogen peroxide on oral carcinogenesis in hamsters. J Periodontol 57: 685-688. [Crossref]

23. Shannon H, Spencer P, Gross K, Tira D (1993) Characterization of enamel exposed to $10 \%$ carbamide peroxide bleaching agents. Quintessence Int 24: 39-44. [Crossref]

24. Bitter NC (1998) A scanning electron microscope study of the long-term effect of bleaching agents on the enamel surface in vivo. Gen Dent46:84-88. [Crossref]

25. Oltu U, Gürgan S (2000) Effects of three concentrations of carbamide peroxide on the structure of enamel. J Oral Rehabil 27: 332-340. [Crossref]

26. Lahtinen SJ, Jalonen L, Ouwehand AC, Salminen SJ (2007) Specific Bifidobacterium strains isolated from elderly subjects inhibit growth of Staphylococcus aureus.Int $J$ Food Microbiol 117: 125-128. [Crossref]

27. Saito M, Seki M, Iida K, Nakayama H, Yoshida S (2007) A novel agar medium to detect hydrogen peroxide-producing bacteria based on the prussian blue-forming reaction. MicrobiolImmunol 51: 889-892. [Crossref]

28. Hillman JD, Socransky SS (1982) Bacterial interference in the oral ecology of Actinobacillusactinomycetemcomitans and its relationship to human periodontosis. Arch Oral Biol 27: 75-77. [Crossref]

29. Gegauff AG, Rosenstiel SF, Langhout KJ, Johnston WM (1993) Evaluating tooth color change from carbamide peroxide gel. J Am Dent Assoc 124: 65-72. [Crossref]

30. Cannon M, Trent B, Vorachek A, Kramer S, Esterly R (2013) Effectiveness of CRT at measuring the salivary level of bacteria in caries prone children with probiotic therapy. J Clin Pediatr Dent 38: 55-60. [Crossref]

31. Hedayati-Hajikand T, Lundberg U, Eldh C, Twetman S (2015) Effect of probiotic chewing tablets on early childhood caries--a randomized controlled trial. BMC Oral Health 15: 112. [Crossref]

Copyright: (C)2015 Hillman JD. This is an open-access article distributed under the terms of the Creative Commons Attribution License, which permits unrestricted use, distribution, and reproduction in any medium, provided the original author and source are credited. 